\title{
Slow magnetoacoustic standing waves in a curved solar coronal slab
}

\author{
R. Ogrodowczyk ${ }^{1}$, K. Murawski ${ }^{2}$, and S. K. Solanki ${ }^{3}$ \\ 1 Department of Computer Sciences, The State School of Higher Education in Chełm, ul. Pocztowa 54, 22-100 Chełm, Poland \\ e-mail: rogrodow@pwsz.chelm.pl \\ 2 Group of Astrophysics and Gravity Theory, UMCS, ul. Radziszewskiego 10, 20-031 Lublin, Poland \\ 3 Max-Planck-Institut für Sonnensystemforschung, Max-Planck-Str. 2, 37191 Katlenburg-Lindau, Germany
}

Received 10 January 2008 / Accepted 1 October 2008

\section{ABSTRACT}

\begin{abstract}
Aims. We consider a model of a two-dimensional solar coronal arcade to explore the effects of a curved magnetic field topology on excitation and attenuation of slow magnetoacoustic standing waves.

Methods. The time-dependent ideal magnetohydrodynamic equations are solved numerically to find the spatial and temporal signatures of these waves.

Results. A pulse in gas pressure initially launched at a loop footpoint excites the fundamental mode of slow magnetoacoustic standing waves. The typical excitation time of such a wave mode is 2.5 wave periods, with a similar attenuation timescale. These values are remarkably similar to those recovered from observations by SOHO/SUMER in an Fe XIX line.

Conclusions. Slow magnetoacoustic standing waves are excited and attenuated more efficiently in curved magnetic field lines than in a straight magnetic slab topology. The waves supported by the magnetic arcade are in far closer agreement with observations.
\end{abstract}

Key words. Sun: corona - Sun: oscillations - Sun: magnetic fields - magnetohydrodynamics (MHD)

\section{Introduction}

Slow magnetoacoustic (henceforth called slow) standing waves have been the subject of intensive observational and theoretical investigations. Observations recovered by the SOHO/SUMER, SOHO/EIT, TRACE/EUV, and YOHKOH/SXT instruments revealed that loop oscillations are triggered impulsively by flares or smaller brightening close to a loop footpoint (De Moortel et al. 2002a,b; Taroyan et al. 2004, 2005; Mariska 2006). In particular, Wang et al. (2005) investigated the excitation of standing slow-mode oscillations and found that develop rapidly, within 1-2 wave-periods of the excitation by a hot impulsive events. It was found by Zaqarashvili et al. (2005) that slow waves may also be excited by the periodic shaking of coronal magnetic field lines due to photospheric motions. Diáz \& Roberts (2006) demonstrated that a structured plasma along the magnetic field lines influence considerably the evolution of slow modes. In another study, Wang et al. (2003a,b) inferred from observational data that slow standing waves are attenuated within a few wave periods. Wave attenuation can result from mechanisms such as: wave leakage into the chromosphere (Ofman 2002; Van Doorsselaere et al. 2004; Ogrodowczyk \& Murawski 2007); lateral wave leakage due to curvature of loops (Roberts 2000); phase mixing (e.g. Ofman \& Aschwanden 2002; Voitenko et al. 2005); resonant absorption (Ruderman \& Roberts 2002); non-ideal magnetohydrodynamic (MHD) effects (Roberts 2000); and electric conduction (Mariska 2006).

Intensive theoretical studies of slow oscillations were completed by Nakariakov et al. (2004) and Tsiklauri et al. (2005), who showed that in a coronal loop an impulsive thermal energy release excites the first harmonic mode efficiently. This result was confirmed by Selwa et al. (2005), who demonstrated that gas pressure pulses launched close to a footpoint excite the fundamental mode of the slow standing wave, while pulses close to the apex trigger the first harmonic. Selwa et al. (2005) performed numerical simulations of slow standing waves in the limit of the one-dimensional approximation. This model was extended to a curved slab by Selwa \& Murawski (2006) and Selwa et al. (2007); who measured a reduction in the excitation time of the fundamental mode in a curved structure in comparison with a straight slab. No-parametric studies were however performed.

The aim of this paper is to generalize the straight magnetic field model of Ogrodowczyk \& Murawski (2007) by taking into account the influence of curvature of magnetic field lines on slow standing waves. The effect of a curved magnetic field topology on fast magnetoacoustic waves was discussed, among others, by Brady et al. (2006), Verwichte et al. (2006), and Terradas et al. (2008). Slow waves are however less well studied. Our goal is to extend the studies of Selwa \& Murawski (2006) and Selwa et al. (2007) by performing appropriate parametric studies of slow standing waves.

This paper is organized as follows. The numerical model is described in Sect. 2. The numerical results are presented in Sect. 3. This paper is then concluded by a short summary of the main results in Sect. 4.

\section{Numerical model}

We describe coronal plasma by the ideal MHD equations:

$\frac{\partial \varrho}{\partial t}+\nabla \cdot(\varrho \boldsymbol{V})=0$

$\varrho \frac{\partial \boldsymbol{V}}{\partial t}+\varrho(\boldsymbol{V} \cdot \nabla) \boldsymbol{V}=-\nabla p+\frac{1}{\mu}(\nabla \times \boldsymbol{B}) \times \boldsymbol{B}$,

$\frac{\partial p}{\partial t}+\nabla \cdot(p \boldsymbol{V})=(1-\gamma) p \nabla \cdot \boldsymbol{V}$

$\frac{\partial \boldsymbol{B}}{\partial t}=\nabla \times(\boldsymbol{V} \times \boldsymbol{B})$,

$\nabla \cdot \boldsymbol{B}=0$, 
where $\varrho$ denotes mass density, $\boldsymbol{V}$ is flow velocity, $\boldsymbol{B}$ is magnetic field, $p$ is gas pressure, $\mu$ is the magnetic permeability, and $\gamma=$ $5 / 3$ is the adiabatic index.

\subsection{Equilibrium configuration}

We adopt the coronal arcade model in which the coronal arcade is settled in a two-dimensional, gravity-free and motionless environment $(\boldsymbol{V}=0)$ (see Oliver et al. 1998, for a detailed description of the initial configuration). In the solar, corona magnetic effects are more significant than gas pressure effects. The pressure gradient, $\nabla p_{\mathrm{e}}$, in the momentum equation Eq. (2) can therefore be neglected, and we can assume that $p_{\mathrm{e}}=$ const. In equilibrium, the Lorentz force must therefore vanish, i.e.

$\frac{1}{\mu}\left(\nabla \times \boldsymbol{B}_{\mathrm{e}}\right) \times \boldsymbol{B}_{\mathrm{e}}=0$.

This equation can be solved by a current-free magnetic field,

$\frac{1}{\mu} \nabla \times \boldsymbol{B}_{\mathrm{e}}=0$.

Henceforth, we limit our discussion to a two-dimensional atmosphere for which the plasma quantities are assumed to be independent of the spatial coordinate $y, \partial / \partial y=0$. As a consequence, Eq. (7) can be satisfied by using the magnetic potential $A(x, z)$,

$\boldsymbol{B}_{\mathrm{e}}=\nabla \times(A \hat{\boldsymbol{y}})$,

where $\hat{\boldsymbol{y}}$ is a unit vector along the $y$-direction and $A(x, z)$ is defined to be

$A(x, z)=B_{0} \Lambda_{\mathrm{B}} \cos \left(x / \Lambda_{\mathrm{B}}\right) \exp \left(-z / \Lambda_{\mathrm{B}}\right)$.

Consequently, the equilibrium magnetic field components are

$B_{\mathrm{ex}}=B_{0} \cos \left(x / \Lambda_{\mathrm{B}}\right) \exp \left(-z / \Lambda_{\mathrm{B}}\right)$,

$B_{\mathrm{ez}}=-B_{0} \sin \left(x / \Lambda_{\mathrm{B}}\right) \exp \left(-z / \Lambda_{\mathrm{B}}\right)$.

Where $B_{0}$ denotes the magnetic field at the reference level $z=0$, and $\Lambda_{\mathrm{B}}=2 L / \pi$ is the magnetic scale-height with $L$ denoting the horizontal half-width of the arcade, which we choose to be $L=100 \mathrm{Mm}$.

We assume that the coronal mass density $\varrho_{\mathrm{c}}$ is constant over the entire spatial region and varies dramatically with $z$ to a photospheric value $\varrho_{\mathrm{ph}}=10^{4} \varrho_{\mathrm{c}}$ at the bottom of the simulation region. The mass density profile $\varrho_{\mathrm{e}}$ is given by

$\varrho_{\mathrm{e}}(z)=\varrho_{\mathrm{c}}\left\{1+\frac{1}{2} d_{\mathrm{ph}}\left[1-\tanh \left(\frac{z}{s_{\mathrm{ph}}}\right)\right]\right\}$,

where $d_{\mathrm{ph}}$ is the ratio of the photospheric mass density, $\varrho_{\mathrm{ph}}$, to the coronal mass density, $\varrho_{\mathrm{c}}$. Symbol $s_{\mathrm{ph}}$ determines the slope of $\varrho_{\mathrm{e}}(z)$ at the boundary to the photosphere-like layer.

We set the sound speed to be $c_{\mathrm{se}}=0.4 \mathrm{Mm} \mathrm{s}^{-1}$ in the ambient coronal medium at the level $z=0$, Alfvén speed $V_{\mathrm{Ae}}=$ $1 \mathrm{Mm} \mathrm{s}^{-1}$, and mass density $\varrho_{\mathrm{c}}=10^{-15} \mathrm{~kg} \mathrm{~m}^{-3}$. Additionally, we choose and hold fixed $s_{\mathrm{ph}}=1.5 \mathrm{Mm}$.

We consider a curved coronal slab embedded in the arcade. The curved slab corresponds to a region of dense plasma. Boundaries of the slab are parallel to magnetic field lines (Fig. 1). The mass density profile across the slab attains the ambient coronal value, $\varrho_{\mathrm{c}}$, as we move away from the slab and is given by

$\varrho(x, z)=\varrho_{\mathrm{c}}+d \varrho_{\mathrm{e}} \exp \left[-\frac{\left(A(x, z)-A_{\mathrm{c}}\right)^{2}}{B_{0}^{2} \sigma^{2}}\right]$,

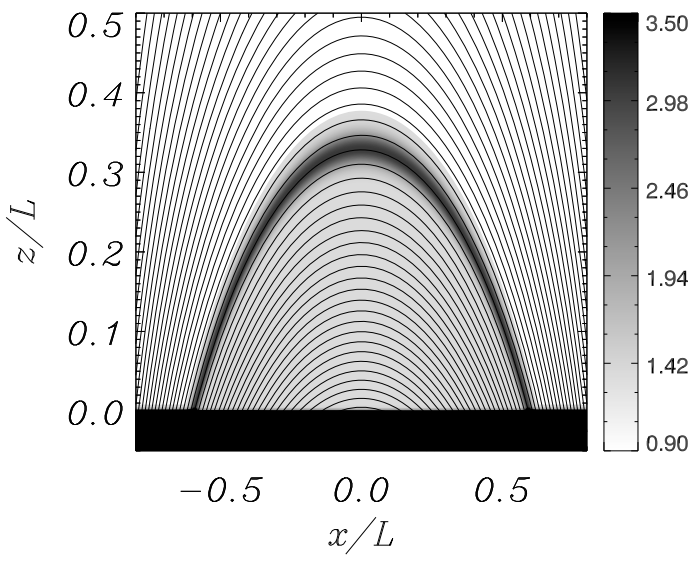

Fig. 1. Initial configuration of the curved slab. Magnetic field lines are indicated by solid lines. The mass density $\varrho$ (color bar) is expressed in arbitrary units $\left(10^{-15} \mathrm{~kg} \mathrm{~m}^{-3}\right)$. The black region below the line $z=0$ corresponds to the photosphere-like layer of dense plasma with a mass density $\varrho_{\mathrm{ph}}=10^{4} \varrho_{\mathrm{c}}$.

where the center of the slab corresponds to a magnetic field line that is defined by $A_{\mathrm{c}}=A\left(L_{\mathrm{f}}, 0\right), \sigma$ is the half-width of the slab, and $d$ denotes the mass density contrast between the slab and the ambient medium. In the simulations, we choose and hold fixed the parameters $d=3$ (Aschwanden \& Nightingale 2005), $\sigma=0.02 L$, and $L_{\mathrm{f}}=0.6 \mathrm{~L}$. For that choice of parameters, the curved slab does not have a purely circular shape. The average main loop radius and length are estimated to be $\sim 60 \mathrm{Mm}$ and $\sim 146 \mathrm{Mm}$, respectively.

\subsection{Perturbations}

The equilibrium state described above can be perturbed in various ways. Since we investigate impulsively excited slow standing waves that are associated with perturbations in gas pressure, at $t=0$ we launch a pulse in gas pressure in the region $x \leq 0$ and $0.0635 L \leq z \leq 0.1 L$, namely

$p(x, z, t=0)=p_{\mathrm{e}}\left\{1+p_{\mathrm{a}} \exp \left[-\frac{\left(A(x, z)-A_{\mathrm{c}}\right)^{2}}{B_{0}^{2} \delta^{2}}\right]\right\}$,

where $p_{\mathrm{a}}$ denotes the relative amplitude of the pulse and $\delta$ is its half-width. We choose $p_{\mathrm{a}}=0.5$ and $\delta=0.02 \mathrm{~L}$. As a result, the pulse is reproduced inside the slab precisely at its left footpoint just above the photosphere (see Eq. (13)). Such a pulse produces local heating within the slab.

\section{Numerical results}

In this section, we present results of the numerical simulations performed. We focus on excitation and attenuation times of slow standing waves that are triggered by the pressure pulse described by Eq. (14). We solve Eqs. (1)-(5) numerically with the code Athena (Gardiner et al. 2005). A higher-order Godunov method is implemented in this code for solving the time-dependent, ideal magnetohydrodynamic equations. A single step, second-order accurate in space and time algorithm is used here. This algorithm is based on the piecewise parabolic method (e.g. Woodward \& Collela 1984). This numerical scheme is supported by a transport scheme that maintains the divergence-free constraint on the magnetic field.

We consider an Eulerian grid with the $x$ - and $z$-dimensions $(-L, L) \times(-0.2 L, 1.3 L)$. This box is covered by a uniform grid of 

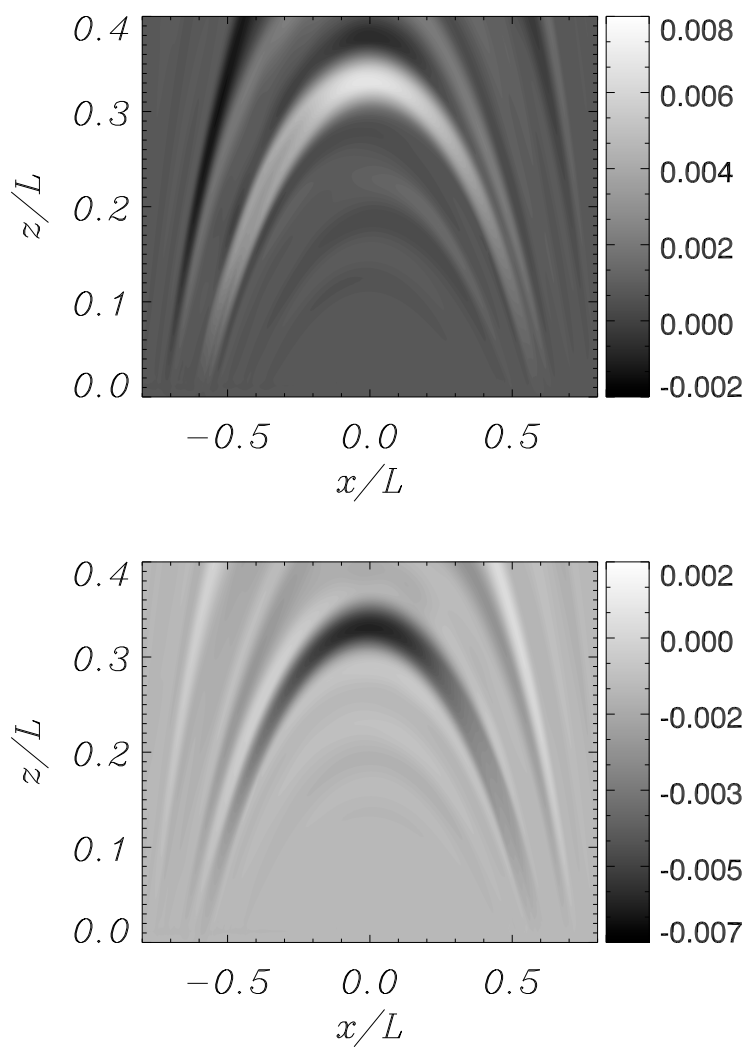

Fig. 2. Spatial profiles of the $x$-component of the velocity, $V_{\mathrm{x}}$, at $t \approx$ $2.25 P_{1}$ (top panel) and at $t \approx 2.75 P_{1}$ (bottom panel) for an initial pulse launched within the slab at its left footpoint. The velocity (color bar) is expressed in arbitrary units of the coronal Alfvén speed, $V_{\mathrm{Ae}}=1 \mathrm{Mm} \mathrm{s}^{-1}$.

$500 \times 500$ numerical cells. We performed grid convergence studies that are based on grid refinement to demonstrate that the numerical results are unaffected by insufficient spatial resolution. We implemented open boundary conditions, with zero-gradient extrapolation for all perturbed plasma quantities at all boundaries of the numerical box. These boundaries enable a wave signal to escape the simulation region completely. However, time invariant boundary conditions for the magnetic field at the bottom and top boundaries of the numerical box are imposed. As described by Eq. (14), we now consider an initial pulse that is launched at the left foot-point of the slab.

Figure 2 displays the $x$-component of the velocity at two moments of time. These panels are approximately in anti-phase and they indicate that the excited oscillation correspond to the fundamental mode, $n=1$, here $n$ denotes the standing wave number such that its wave period, $P_{\mathrm{n}}$, can be evaluated from the analytical formula

$P_{\mathrm{n}} \simeq \frac{2 l}{n c_{\mathrm{Ti}}}, \quad n=1,2, \ldots$,

where $c_{\mathrm{Ti}}=c_{\mathrm{Si}} V_{\mathrm{Ai}} / \sqrt{c_{\mathrm{si}}^{2}+V_{\mathrm{Ai}}^{2}}$ is the tube speed within the slab and $l$ denotes the slab's length. Sound, $c_{\mathrm{si}}$, and Alfvén, $V_{\mathrm{Ai}}$, speeds are evaluated for the curved slab equilibrium to be

$c_{\mathrm{si}}=\sqrt{\frac{\gamma p_{\mathrm{e}}}{d \varrho_{\mathrm{e}}}}, \quad V_{\mathrm{Ai}}=\frac{B_{0}}{\sqrt{\mu d \varrho_{\mathrm{e}}}}$.

In our approach, a value of the tube speed corresponds to the mass density ratio, $d \cong\left(c_{\mathrm{se}} / c_{\mathrm{Ti}}\right)^{2} \cong 2.2$, at the slab apex. These

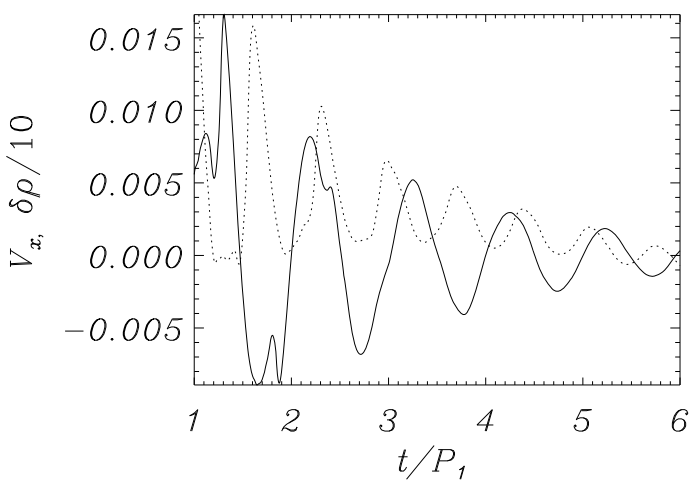

Fig. 3. Time-signatures of velocity $V_{\mathrm{x}}$ (solid line) and relative density enhancement $\delta \varrho$ (dotted line), evaluated at the slab apex for an initial pulse launched within the curved slab at its left footpoint. Here $V_{\mathrm{x}}$, time $t$, and $\delta \varrho$ are expressed in units of $V_{\mathrm{Ae}}=1 \mathrm{Mm} \mathrm{s}^{-1}$, fundamental period, $P_{1}$, and density outside the loop, $\varrho_{\mathrm{e}}$ respectively.

findings confirm earlier claims by Selwa et al. (2005), Selwa \& Murawski (2006), and Selwa et al. (2007) that an initial pulse launched close to a footpoint excites a fundamental mode.

The fundamental slow standing wave produces timesignatures of $V_{\mathrm{x}}$ that can be visualized at any particular spatial location. Figure 3 displays $V_{\mathrm{x}}(t)$ (solid line) and the perturbed filtered mass density $\delta \varrho(t)$ (dotted line), at the apex of the curved slab. We note the phase-shift between $V_{\mathrm{x}}$ and $\delta \varrho$. Nakariakov and Verwichte (2005) showed that for a slow standing wave the parallel component of the velocity experiences a quarter wave-period phase-shift compared with perturbations in mass density $\delta \varrho$.

We use an excitation criterion (Selwa et al. 2005) to find out the excitation time $t_{\text {ex }}$ of slow standing waves in a physical system. According to this criterion the fundamental standing wave is excited at $t_{\mathrm{ex}}>2.5 P_{1}$ (see also Fig. 2). It is noteworthy that this value of $t_{\mathrm{ex}}$ is smaller than in the one-dimensional model of Selwa et al. (2005) and in the straight slab case of Ogrodowczyk $\&$ Murawski (2007). This value of $t_{\mathrm{ex}}$ is approximately twice that in the curved slab case discussed by Selwa \& Murawski (2006) and Selwa et al. (2007). The reason for this discrepancy lies either in the presence of fast magnetoacoustic waves in a two-dimensional system (Selwa \& Murawski 2006) or in the wide pulse used by Selwa \& Murawski (2006) and Selwa et al. (2007) that triggers wave signals both within the curved slab and in the ambient medium. A pulse signal in the ambient medium is also associated with fast magnetoacoustic waves that are able to spread wave energy on a shorter timescale than slow waves. As a consequence, the energy of the initial pulse is spread over the slab structure more rapidly than by slow waves alone, and slow standing waves are sustained over a smaller timescale.

From Fig. 3, we infer that by $t \geq 4 P_{1}$ the wave signal is any longer that of a standing wave. This detuning between the oscillations in mass density and parallel component of velocity was already observed but not reported by Selwa et al. (2005) and Ogrodowczyk \& Murawski (2007). This detuning may result from nonlinear effects, which, after a long time start to play a role. It is also interesting that the perturbed mass density $\delta \varrho(t)=$ $\varrho(t)-\varrho(t=0)>0$ retains its positive values. This behavior is a result of inflow of additional material to the apex from the initial pulse. This inflow results in a permanent evacuation of plasma at the region in which the pulse was launched.

Figure 4 displays the Fourier power spectrum of $V_{\mathrm{x}}$ evaluated at the slab apex (Fig. 3). This spectrum confirms the 


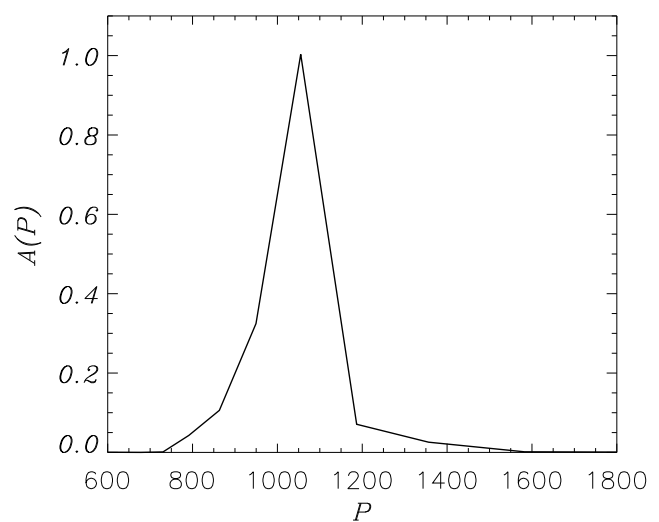

Fig. 4. Fourier power spectrum of the slow wave signal collected at the slab apex. The amplitude of the power spectrum $A(P)$ is normalized to 1 . Wave period $P$ is given in seconds.

presence of the fundamental mode, $n=1$, with wave period $P_{1} \cong 1054 \pm 30 \mathrm{~s}$.

To estimate the attenuation time, $\tau$, of a slow standing wave, we fit the curve

$V_{\mathrm{x}}(t)=V_{\mathrm{x} 0} \exp \left(-\frac{t-t_{\mathrm{ex}}}{\tau}\right)$

to the time-signature of $V_{\mathrm{x}}$ (Fig. 3), where $V_{\mathrm{x} 0}$ denotes the amplitude of $V_{\mathrm{x}}$ at the excitation time $t_{\mathrm{ex}}$ and $V_{\mathrm{x} 0}$ is used as an initial parameter entering the fitting subroutine. In this way, we find that $\tau \cong 1.85 P_{1}$ for the fundamental standing wave, $n=1$.

To investigate the excited standing mode, we adopt the flux coordinate system (Oliver et al. 1996)

$\psi=\cos \left(x / \Lambda_{\mathrm{B}}\right) \exp \left(-z / \Lambda_{\mathrm{B}}\right)$

$\chi=\sin \left(x / \Lambda_{\mathrm{B}}\right) \exp \left(-z / \Lambda_{\mathrm{B}}\right)$.

Flux coordinates $\chi$ and $\psi$ are in directions that are parallel and perpendicular to the curved slab respectively. In the system $(\chi, \psi)$, the curved slab becomes straight (Fig. 5). We note that the photosphere-like layer is at the left and right of Fig. 5 and the slab runs from left to right.

Figure 5 illustrates the spatial profiles of $p(\chi, \psi)$ at $t \simeq$ $2.75 P_{1}$ (top panel), $p(x=0, z)$ (middle panel), and along the slab, for $\psi=0.4355$, (bottom panel). In agreement with Fig. 2 , we are able to discern that wave energy is also present in the ambient medium, both above (for $z / L>0.4$ ) and below the loop apex (for $z / L<0.2$ ) (middle panel). We note that part of the energy leaks into the photosphere-like layer, $|\chi|>0.5$, giving rise to a prominent pattern of maxima and minima. These wave fronts agree well with the picture in which oscillation within the loop decays with time. In the corona, the wavelength of waves leaking through the footpoints is $\lambda_{\mathrm{i}} \cong 2.89 \mathrm{~L}$. In the photospherelike layer, we have $\lambda_{\mathrm{ph}} \cong 0.0421 L . \lambda_{\mathrm{i}} / \lambda_{\mathrm{ph}}=68.6$, which is close to $c_{\mathrm{Ti}} / c_{\mathrm{Tph}}=67.5$. These values satisfy the relations $\lambda_{\mathrm{i}}=c_{\mathrm{Ti}} P_{1}$ and $\lambda_{\mathrm{ph}}=c_{\mathrm{Tph}} P_{1}$. As a result, we infer that waves reduce their wavelength, while leaking into the photosphere-like layer, due to the low slow-mode speed there (the wave has managed to propagate only slightly during the wave period). This is what we can see in Fig. 5.

At the photosphere-like layer, slow waves become partially reflected back into the top medium The wave reflection coefficient can be defined to be (LeVeque 2002, Eq. (9.52))

$R=\frac{Z_{\mathrm{ph}}-Z_{\mathrm{i}}}{Z_{\mathrm{i}}+Z_{\mathrm{ph}}}$
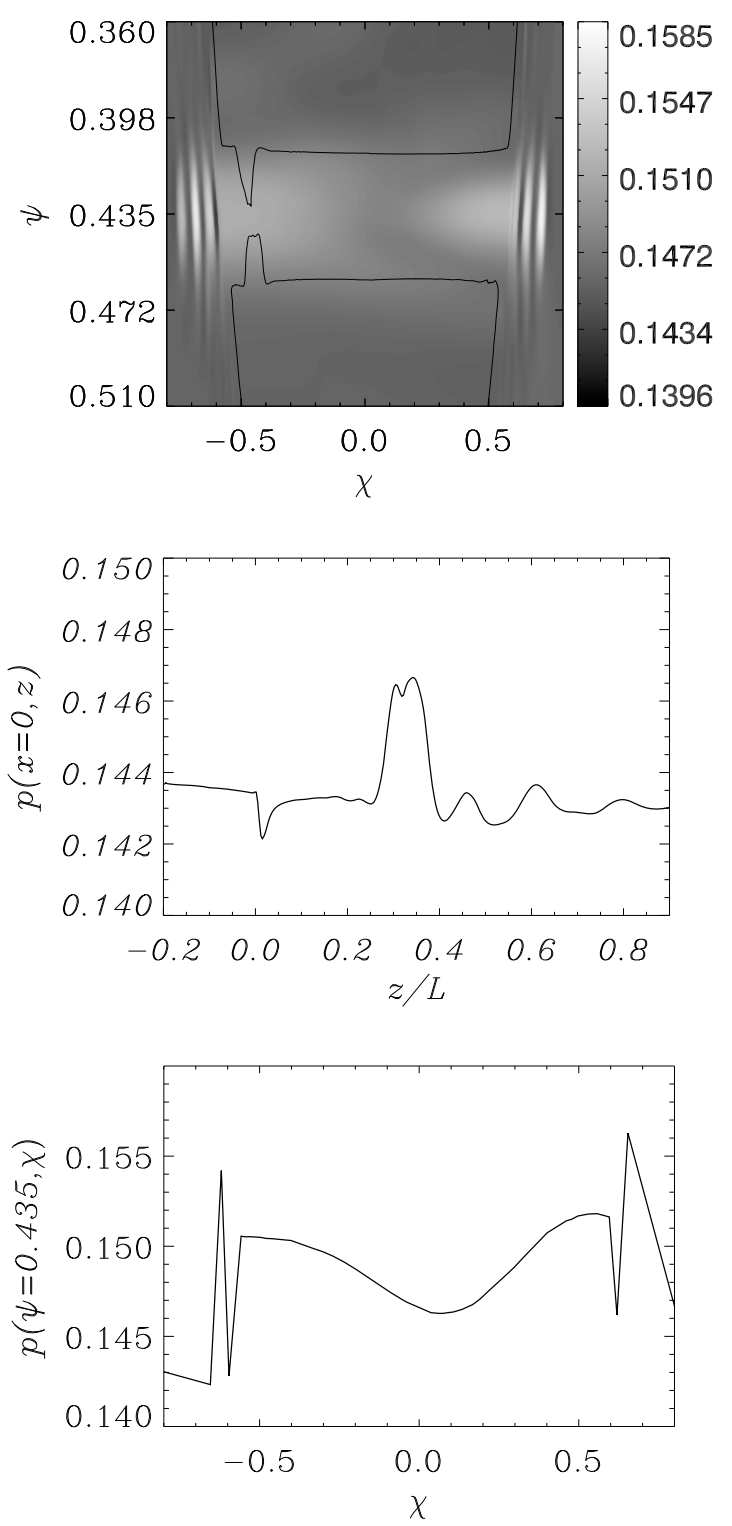

Fig. 5. Spatial distribution of gas pressure (top panel) and its slices along $x=0$ (middle panel) and $\psi=0.4355$ (bottom panel). The solid lines (top panel) illustrate the approximate location of the curved slab (horizontal lines) and the interface between the solar corona and photosphere-like layer (vertical lines), presented in flux coordinates (Eqs. (18) and (19)).

where $Z_{\mathrm{ph}}=\varrho_{\mathrm{ph}} c_{\mathrm{Tph}}$ and $Z_{\mathrm{i}}=\varrho_{\mathrm{i}} c_{\mathrm{Ti}}$ denote the impedance of the photosphere-like layer and the slab, respectively. We estimate that $Z_{\mathrm{ph}}=40$ and $Z_{\mathrm{i}}=0.231$, which provides $R=0.98$. Thus, $98 \%$ of each component of the incident wave will reflect every time it collides with the interface and $2 \%$ will be transmitted. This finding is in qualitative agreement with the numerical results plotted in the bottom panel of Fig. 5 .

We now consider the averaged energy density of slow waves

$\hat{E}(t)=\frac{1}{S} \int_{\psi_{1}}^{\psi_{2}} \int_{\chi_{1}}^{\chi_{2}} E(\chi, \psi, t) \mathrm{d} \chi \mathrm{d} \psi$

where $S$ is the spatial area over which the integration is performed, and $\psi_{1}$ and $\psi_{2}\left(\chi_{1}\right.$ and $\left.\chi_{2}\right)$ are the limits of the integration along the $\psi-\left(\chi^{-}\right)$direction. The energy density, $E(\chi, \psi)$ 

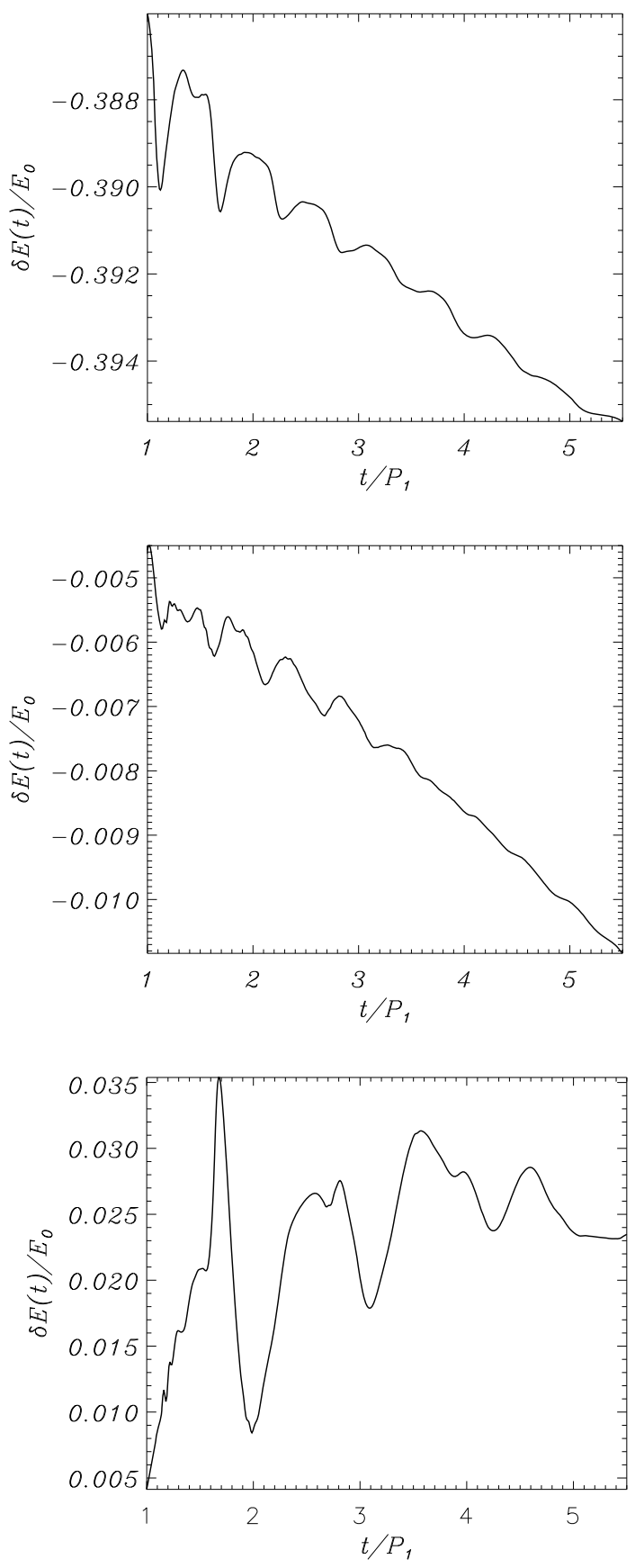

Fig. 6. Evolution of the perturbed energy computed from Eq. (21) within the slab $(0.41 \leq \psi \leq 0.45)$ (top panel), above the slab $(0.34 \leq$ $\psi \leq 0.38$ and $-0.45 \leq \chi \leq 0.45$ ) (middle panel), and inside the photosphere-like layer $(0.41 \leq \psi \leq 0.45$ and $0.55 \leq \chi \leq 0.8)$ (bottom panel).

is expressed as the sum of kinetic and internal energy densities, namely

$E(\chi, \psi, t)=\frac{\varrho V^{2}}{2}+\frac{p}{\gamma-1}$.

The top panel of Fig. 6 illustrates the evolution in the normalized energy, $\delta \hat{E}(t) / E_{0}=(\hat{E}(t)-\hat{E}(t=0)) / \hat{E}(t=0)$, evaluated within the slab $(0.41 \leq \psi \leq 0.46)$. It is clear that energy leakage is also present during the initial transient stage $\left(t<1 P_{1}\right)$. For $2 \leq t / P_{1} \leq 5.6$, the energy decreases more rapidly with in time than in the straight slab case (not shown). As a result,

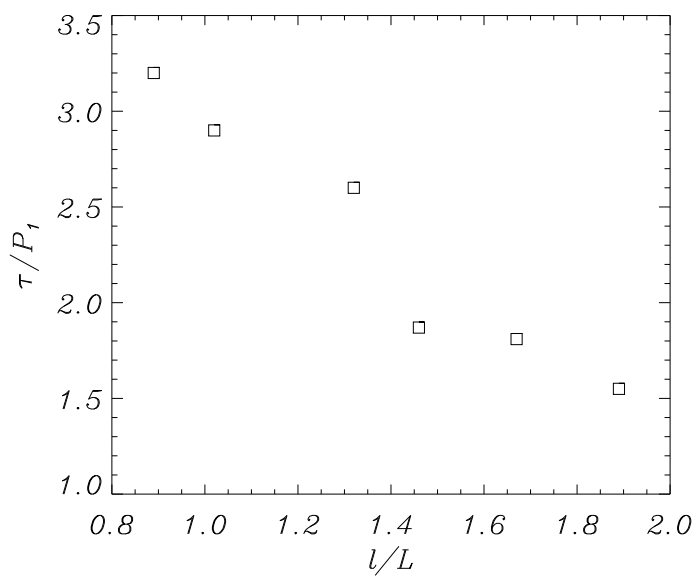

Fig. 7. Normalized standing slow wave attenuation time $\tau / P_{1}$ versus normalized loop length $l / L$.

we conclude that it is caused by a curved magnetic field, which results in energy leakage to the ambient corona across the slab edges (middle panel of Fig. 5). It is noteworthy that energy decreases about twice as rapidly within the slab (Fig. 6, top panel) as above the slab (middle panel). We infer that the energy leaks to both the ambient corona and the photosphere-like layer (bottom panel). As a result of energy penetrating the photospherelike layer, $\delta \hat{E}(t) / E_{0}$ is there positive. Due to energy leakage from the slab, $\delta \hat{E}(t) / E_{0}$ is negative within the slab. We also discovered that $\delta \hat{E}(t) / E_{0}<0$ in the region above the slab and a physical reason for this is presently unclear.

We now present the results of parametric studies that we performed. For these studies, we changed the curvature of the loop by varying the positions of its footpoints. This can be achieved by varying Ac in Eq. (13). We note that a longer loop exhibits a larger curvature of magnetic field lines (Fig. 1). This approach enabled us to determine the influence of the magnetic-field-line curvature on the attenuation time of slow standing waves.

Figure 7 show a plot of the normalized standing wave attenuation time $\tau / P_{1}$ versus normalized loop length $l / L$. We note that $\tau / P_{1}$ decreases with $l / L$. As a result, we conclude that more strongly curved loops result in shorter attenuation times. One scenario to explain this dependence is that it is a consequence of energy leakage through slab edges to the ambient corona. According to this scenario, energy leakage is more significant for longer slabs, for which have the magnetic field lines stronger curvature. On the other hand, if magnetic field lines are longer and the width of the initial pressure pulse remains constant, then there will be more power at shorter wavelengths. These shorter waves are not reflected from the dense layers and the slow wave therefore attenuates more rapidly by means of leakage into the photosphere.

\section{Summary}

We have developed a two-dimensional model of a curved coronal slab resting on a dense photosphere-like layer in performing numerical simulations of standing slow magnetoacoustic waves. These waves are triggered by a pressure pulse that is initially launched within the slab. Such a pulse generates fundamental slow modes, in good agreement with the results of Selwa et al. (2005). These slow waves leak their energy into both the photosphere-like layer and the ambient coronal medium. Our 
numerical results show that curvature of magnetic field lines affects significantly the excitation and attenuation of slow standing waves. As a result of energy leakage through the slab edges at the apex of a curved loop, the standing slow waves are sustained and attenuated more rapidly than in a straight slab (Selwa et al. 2005).

Observational findings (Wang et al. 2002, 2003a,b, 2005) revealed that slow standing waves are quickly excited and strongly attenuated within 1-2 waves periods and have typical periods of 14-18 min. These findings corresponded to a relatively long loop $(l=191 \mathrm{Mm})$ and high sound speed $c_{\mathrm{s}}=380 \mathrm{~km} \mathrm{~s}^{-1}$ (Wang et al. 2003a). In our model, we find that the fundamental mode possesses a wave period equal to $17.5 \mathrm{~min}$ and the excitation and decay times were estimated to be $t_{\mathrm{ex}}=2.5 P_{1}$ and $\tau=1.85 P_{1}$, respectively. These results correspond to $l=146 \mathrm{Mm}$ and sound speed within the curved slab of $c_{\mathrm{si}}=0.231 \mathrm{Mm} \mathrm{s}^{-1}$. It is gratifying that by implementing curved magnetic field lines such good agreement with observations could be achieved (Wang et al. 2002, 2003a,b). This agreement was far closer than achieved by either the one-dimensional model of Selwa et al. (2005) or the two-dimensional straight slab model of Ogrodowczyk \& Murawski (2007). Selwa et al. (2005) found that the ratio of attenuation time $\tau$ to wave period $P_{1}$ was $\tau / P_{1} \approx 5$. Ogrodowczyk $\&$ Murawski (2007) measured a ratio of $\tau / P_{1} \approx 3.8$, while the present model reveals that $\tau / P_{1}$ can be as low as 1.85 for a loop of length $l=143 \mathrm{Mm}$.

Acknowledgements. The authors express their thanks to the referee for his/her helpful comments. R.O.'s and K.M.'s work was supported by a grant from the State Committee for Scientific Research Republic of Poland, with MNiI grant for years 2007-2010. R.O. expresses his thanks to Dr. M. Selwa for her assistance with data visualization in IDL. Part of this work was completed during a visit by R.O. to the Max-Planck-Institut für Sonnensystemforschung in Katlenburg-Lindau. The magnetohydrodynamic code used in this study was developed at Princeton University by Tom Gardiner, Jim Stone, Peter Teuben, and John Hawley with the support of the NSF Information Technology Research (ITR) program.

\section{References}

Aschwanden, M. J., \& Nightingale, R. W. 2005, ApJ, 633, 499 Brady, C. S., Verwichte, E., \& Arber, T. D. 2006, A\&A, 449, 389

Diáz, A. J., \& Roberts, B. 2006, A\&A, 458, 975

De Moortel, I., Hood, A. W., \& Ireland, J. 2002a, A\&A, 381, 311

De Moortel, T., Hood, A. W., Ireland, J., \& Walsh R.W. 2002b, Sol. Phys., 209, 89

Gardiner, T. A., \& Stone, J. M. 2005, J. Comput. Phys., 205, 509

LeVeque, R. J. 2002, Finite-Volume Methods for Hyperbolic Problems (Cambridge University Press)

Mariska, J. T. 2006, ApJ, 639, 484

Nakariakov, V. M., Tsiklauri, D., Kelly, A., Arber, T. D., \& Aschwanden, M. J. 2004, A\&A, 414, 25

Nakariakov, V. M., \& Verwichte, E. 2005, Living Rev. Sol. Phys., 2, 3

Ofman, L. 2002, ApJ, 568, L135

Ofman, L., \& Aschwanden, M. 2002, ApJ, 576, L153

Ogrodowczyk, R., \& Murawski, K. 2007, A\&A, 467, 311

Oliver, R., Hood, A., \& Priest, E. R. 1996, ApJ, 461, 224

Oliver, R., Murawski, K., \& Ballester, J. L. 1998, A\&A, 330, 726

Roberts, B. 2000, Sol. Phys., 193, 139

Ruderman, M. S., \& Roberts, B. 2002, ApJ, 577, 475

Selwa, M., \& Murawski, K. 2006, in Proceedings of SOHO 17 - 10 Years of SOHO and Beyond, Ed. H. Lacoste, ESA SP-617, 76.1

Selwa, M., Murawski, K., \& Solanki, S. K. 2005, A\&A, 435, 701

Selwa, M., Ofman, L., \& Murawski, K. 2007, ApJ, 668, L83

Taroyan, Y., Erdélyi, R., \& Doyle, J. G. 2004, Proceedings SOHO 15 Workshop - Coronal Heating, ed. R. W. Walsh, J. Ireland, D. Danesy, \& B. Fleck (Paris: European Space Agency), ESA SP-575, 443

Terradas, J., Oliver, R., \& Ballester, J. L. 2008, ApJ, 675, 875

Taroyan, Y., Erdélyi, R., Doyle, J. G., \& Bradshaw, S. J. 2005, A\&A, 438, 713

Tsiklauri, D., Nakariakov, V. M., Arber, T. D., \& Aschwanden, M. J. 2004, A\&A, 422,351

Van Doorsselaere, T., Debosscher, A., Andries, J., \& Poedts, S. 2004, A\&A, 424, 1065

Verwichte, E., Foullon, C., \& Nakariakov, V. M. 2006, A\&A, 446, 1139

Wang, T. J., Solanki, S. K., Curdt, W., Innes, D. E., \& Dammasch, I. E. 2002, ApJ, 574, L101

Wang, T. J., Solanki, S. K., Innes, D. E., Curdt, W., \& Marsch, E. 2003a, A\&A, 402, L17

Wang, T. J., Solanki, S. K., Curdt, W., et al. 2003b, A\&A, 406, 1105

Wang, T. J., Solanki, S. K., Innes, D. E., \& Curdt, W. 2005, A\&A, 425, 753

Voitenko, Y., Andries, J., Copil, P. D., \& Goossens, M. 2005, A\&A, 437, L47

Woodward, P., \& Collela, P. 1984, J. Comput. Phys., 54, 115

Zaqarashvili, T. V., Oliver, R., \& Ballester, J. L. 2005, A\&A, 433, 357 